\title{
Successful rechallenge with reduced dose of erlotinib in a patient with lung adenocarcinoma who developed erlotinib-associated leukocytoclastic vasculitis: A case report
}

\author{
BO-AN SU ${ }^{1}$, WAN-LIN SHEN ${ }^{2}$, SHENG-TSUNG CHANG $^{3}$, LI-YIA FENG $^{4}$, CHIA-JUNG WU $^{5}$ and YIN-HSUN FENG ${ }^{6,7}$ \\ Divisions of ${ }^{1}$ Infection, and ${ }^{6}$ Hematology and Oncology, Department of Internal Medicine; ${ }^{3}$ Department of Pathology, \\ Chi-Mei Medical Center, Yong Kang, Tainan 71004; Departments of ${ }^{2}$ Pathology, and ${ }^{5}$ Nursing, Chi-Mei Medical Center, \\ Liouying, Tainan 73657; ${ }^{4}$ National Kaohsiung University of Hospitality and Tourism, Xiaogang, Kaohsiung 81271; \\ ${ }^{7}$ College of Medicine and Life Science, Chung Hwa University of Medical Technology, Tainan 71755, Taiwan, R.O.C.
}

Received January 5, 2012; Accepted March 5, 2012

DOI: $10.3892 / 01.2012 .647$

\begin{abstract}
The oral tyrosine kinase inhibitors of epidermal grow th factor, erlotinib and gefitinib, are active in the treatment of non-small cell lung cancer (NSCLC). However, a number of skin manifestations have been found in patients receiving erlotinib therapy. Leukocytoclastic vasculitis is a rare sideeffect of erlotinib therapy. However, whether or not erlotinib treatment should be continued when disseminated ulceration of leukocytoclastic vasculitis is encountered remains to be determined. In this study, we report a patient with NSCLC who remains responsive to erlotinib treatment following successful rechallenge with a reduced dose of erlonitib after presenting with severe degree of leukoclastic vasculitis.
\end{abstract}

\section{Introduction}

Lung cancer is the leading cause of cancer-related mortality in males and females. Advances in the knowledge of tumor biology and the mechanisms of oncogenesis have led to the selection of several molecular targets for lung cancer treatment. The epidermal growth factor receptor (EGFR) has received particular attention for lung cancer treatment. EGFR is a transmembrane receptor found on cells of epithelial origin, which is commonly expressed at a high level in a variety of solid tumors. It is involved in the control of cell proliferation, metastasis and angiogenesis (1). The therapeutic inhibition of EGFR with monoclonal antibodies to antagonize ligandreceptor binding or small-molecules to inhibit tyrosine kinase domain activation, is the main pharmacological strategy in clinical development for therapeutics. Erlotinib, an orally

Correspondence to: Dr Yin-Hsun Feng, Division of Hematology and Oncology, Department of Internal Medicine, Chi-Mei Medical Center, 901 Chung-Hwa Road, Yong Kang, Tainan 71004, Taiwan, R.O.C.

E-mail: yinhsun.feng@gmail.com

Key words: erlotinib, leukocytoclastic vasculitis, lung cancer available inhibitor of EGFR tyrosine kinase, has been shown to improve survival in non-small cell lung cancer (NSCLC) patients following first- or second-line chemotherapy. However, various skin manifestations have been reported in patients with NSCLC who received erlotinib therapy. Skin reactions are the most common adverse events associated with erlotinib, and generally develop within 7-10 days of treatment initiation. Clinical manifestations include papulopustular rash, xerosis, paronychia and hair changes (2). Skin rash may spontaneously resolve and reappear, and is reversible following drug discontinuation. However, when it develops, the chronic side-effect is extremely distressing for patients. We report a patient with metastatic lung cancer, who was diagnosed with histologically confirmed cutaneous leukocytoclastic vasculitis during erlotinib treatment. After a short period of erlotinib withdrawal, leukocytoclastic vasculitis was not reproduced following successful restoration of a reduced dose of erlotinib.

Approval for the study was obtained from the Institutional Review Board of the Chi-Mei Medical Center, Tainan, Taiwan. Patient consent was obtained.

\section{Case report}

The patient was a 50-year-old female with metastatic lung adenocarcinoma, who had initial symptoms of multiple bone pain and a cough. The diagnosis of lung cancer was determined from the biopsied pathology from bone metastasis (Fig. 1), and a computed tomography of the chest. The patient initiated first-line treatment with erlotinib (150 mg daily) and bevacizumab $(15 \mathrm{mg} / \mathrm{kg}$ every 3 weeks) in September 2008. Following eight days of treatment with erlotinib and bevacizumab, the patient presented with papulopustules over the scalp, face, trunk and extremities. Topical steroids and antibiotics were administered to alleviate her discomfort. Five weeks later, purpuric lesions surrounding the red areas of necrosis were located mostly at the extremities. The patient's skin reactions progressed to extensive ulcerations (Fig. 2A). A skin biopsy was performed on the patient's leg four months after the initiation of erlotinib, which revealed an infiltration of neutrophils and red blood cells surrounding the vessels in the upper dermis (Fig. 2B). 
A
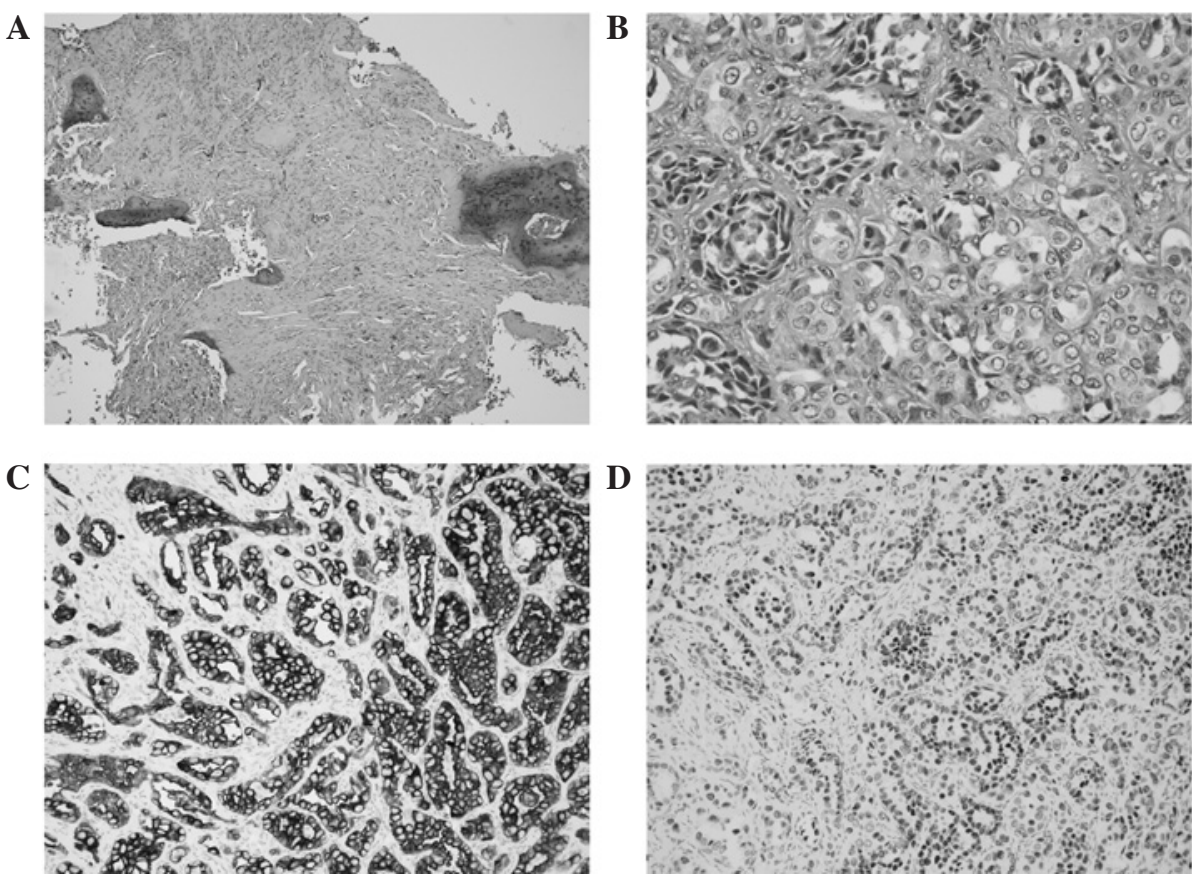

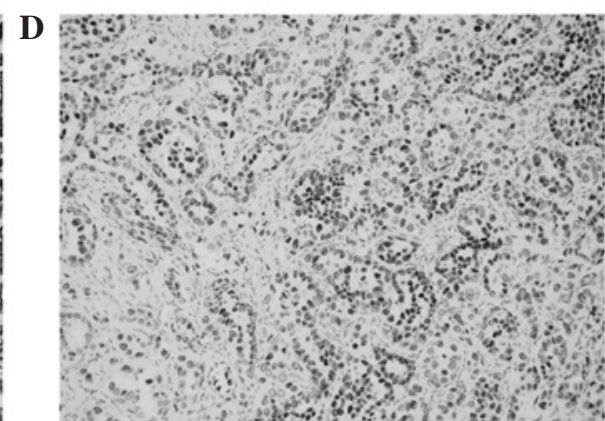

Figure 1. Lung cancer pathology. Excisional biopsy of the left femur at diagnosis revealed moderately differentiated adenocarcinoma at (A) low-power field (H\&E; original magnification, x100) and (B) high-power field (H\&E; original magnification, x400). The tumor cells expressed (C) immunochemically-positive cytokeratin-7 staining (original magnification, x200) and (D) positive thyroid transcription factor-1 staining (original magnification, x200).

A

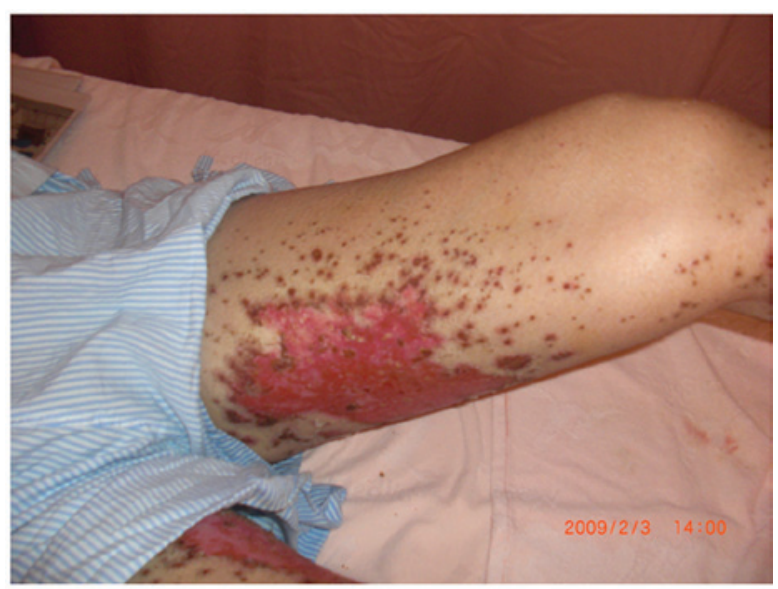

B

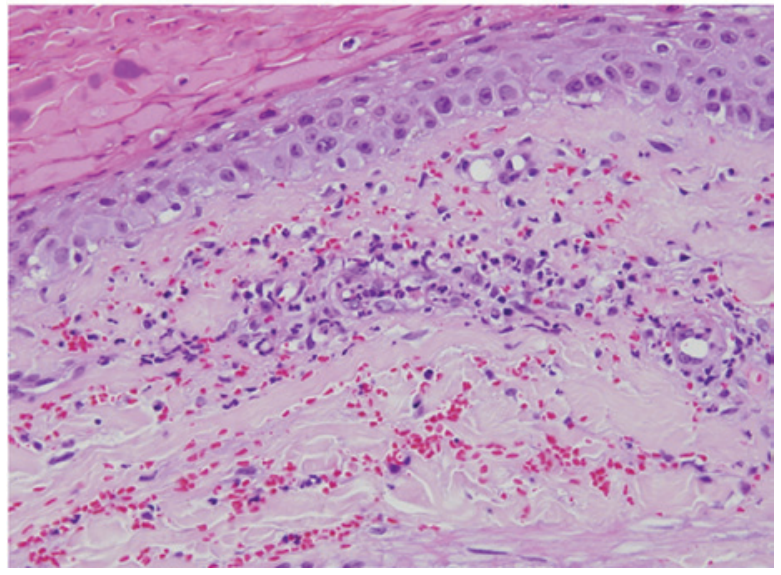

Figure 2. (A) Purpuric lesions surrounded extensive ulceration in a patient treated with erlotinib. (B) The upper dermal small vessels revealed neutrophil-rich infiltration associated with extravasation of erythrocytes in the adjacent tissue. (H\&E; original magnification, $\mathrm{x} 400)$.

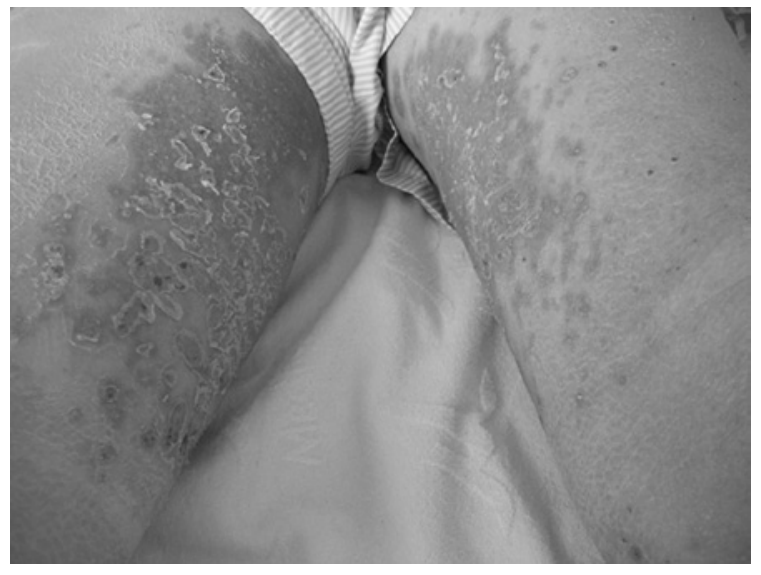

Figure 3. A total of 10 days after discontinuation of erlotinib, the lesions healed without ulceration in the patient.

A histological diagnosis of leukocytoclastic vasculitis was determined based on these findings. Erlotinib was discontinued one week after the skin biopsy without interruption of bevacizumab. The skin ulceration healed 10 days after the discontinuation of erlotinib (Fig. 3), and resolved completely seven weeks later. Erlotinib at a dose of $100 \mathrm{mg}$ daily was challenged in this patient after almost complete resolution of the skin reaction. The patient's lung malignancy remains responsive to the reduced dose of erlotinib and bevacizumab more than 2 years later, without recurrence of skin ulcerations (Fig. 4).

\section{Discussion}

Among skin manifestations associated with erlotinib therapy, acneiform eruption usually consisting of follicular papules 
A
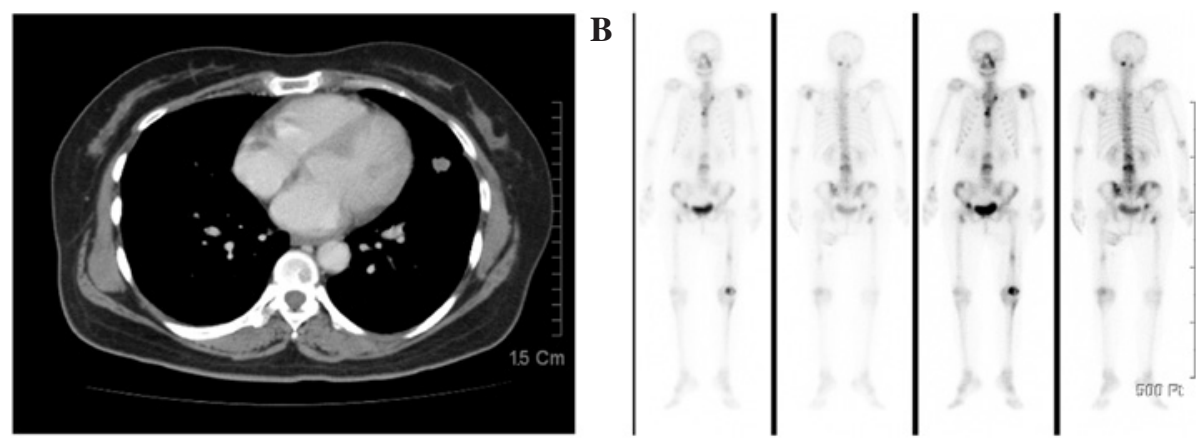

C

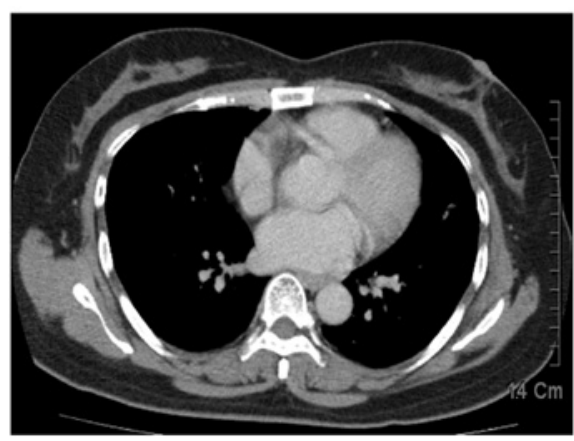

D

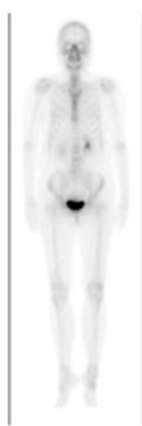

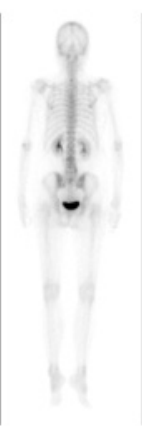
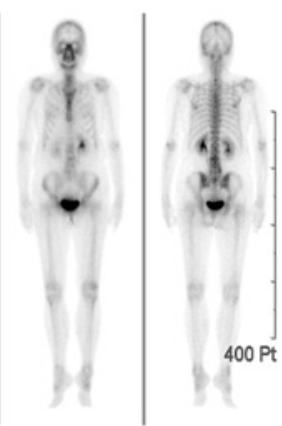

Figure 4. Image shows tumors before treatment of erlotinib and bevacizumab. (A) A lung nodule $1.5 \mathrm{~cm}$ in size located at the left upper lobe of the lung as detected by computed tomography of the chest. (B) The bone scan revealed uptake of radionuclide at the sternum, lumbar spine and left shoulder. Two years after treatment $(\mathrm{C})$ regression of the lung nodule was detected by computed tomography of the chest and (D) decreasing radionuclide uptake of bone metastasis revealed by the bone scan.

and pustules without comedones or propionibacterium acne is observed in more than $90 \%$ of patients (3). Vasculitis is a histological diagnosis defined as inflammation targeting the blood vessel walls, which compromises their function, leading to hemorrhagic or ischemic events. Etiologically, vasculitis can be separated into primary or secondary vasculitis following the determination of offending factors, including connective tissue disease, infection, adverse drug reaction or a paraneoplastic phenomenon.

Inflammation of the small blood vessels, most commonly postcapillary venules, is the cardinal histological feature of leukocytoclastic vasculitis. Other characteristic features include fibrinoid necrosis of the vessel walls, leukocytoclastosis and hemorrhage. The inflammatory infiltrate is typically neutrophilic; other studies have demonstrated a predominance of mononuclear cells and eosinophils distributed in all vessel layers (4). The skin is the most commonly involved organ in leukocytoclastic vasculitis, predominantly in the lower extremities. Up to one-third of patients have trunk and upper extremity involvement, typically sparing the palmar, plantar and mucosal surfaces. Leukocytoclastic vasculitis is rarely observed in patients treated with erlotinib. Boeck et al have reported two patients treated with erlotinib who developed hemorrhagicnecrotic type leukocytoclastic vasculitis (5). None of these reported patients were challenged with erlotinib again. However, Asian patients with lung adenocarcinoma have been reported to benefit from erlotinib therapy (6). Concerning the incidence of erlotinib-related skin rash, data from a phase III randomized clinical trial of erlotinib versus a placebo in pretreated NSCLC patients revealed that rash developed in $75 \%$ of patients who received erlotinib, but only $1 \%$ definitively discontinued erlo- tinib therapy (BR.21 trial, conducted by NCIC CTG (National Cancer Institute of Canada Clinical Trials Group).

It is very difficult to determine the permanent withdrawal of erlotinib, particularly when patients benefit from erlotinib. Skin rash may spontaneously resolve and reappear during erlotinib discontinuation. The chronic side effect of skin rash is extremely distressing for patients. However, whether or not to continue erlotinib therapy when facing this clinical challenge has yet to be determined. This is the first report to assess whether to rechallenge with erlotinib when encountering a severe degree of leukocytoclastic vasculitis. To ensure the safety of a lower dose of erlotinib in erlotinib-associated leukocytoclastic vasculitis, we therefore strongly suggest that preclinical and clinical investigations of this syndrome associated with erlotinib are significant in making clinical decisions.

\section{References}

1. Ciardiello F, De Vita F, Orditura M, De Placido S and Tortora G: Epidermal growth factor receptor tyrosine kinase inhibitors in late stage clinical trials. Expert Opin Emerg Drugs 8: 501-514, 2003.

2. Segaert S and Van Cutsem E: Clinical signs, pathophysiology and management of skin toxicity during therapy with epidermal growth factor receptor inhibitors. Ann Oncol 16: 1425-1433, 2005.

3. Segaert S, Tabernero J, Chosidow O, et al: The management of skin reactions in cancer patients receiving epidermal growth factor receptor targeted therapies. J Dtsch Dermatol Ges 3: 599-606, 2005.

4. Carlson JA: The histological assessment of cutaneous vasculitis. Histopathology 56: 3-23, 2010.

5. Boeck S, Wollenberg A and Heinemann V: Leukocytoclastic vasculitis during treatment with the oral EGFR tyrosine kinase inhibitor erlotinib. Ann Oncol 18: 1582-1583, 2007.

6. Kubota K, Nishiwaki Y, Tamura T, et al: Efficacy and safety of erlotinib monotherapy for Japanese patients with advanced non-small cell lung cancer: a phase II study. J Thorac Oncol 3: $1439-1445,2008$. 